\title{
REDES UNIVERSITARIAS EN COLOMBIA. NUEVA CONCEPCIÓN HISTÓRICA PARA LA UNIVERSIDAD
}

\author{
Diana Elvira Soto Arango ${ }^{1}$ \\ Universidad Pedagógica y Tecnológica de Colombia - RUDECOLOMBIA \\ Grupo de Investigación - HISULA \\ dianaelvirasoto@gmail.com
}

Recepción: 22/09/2010

Evaluación: $12 / 10 / 2010$

Aceptación: 18/10/2010

Artículo de Reflexión

\section{RESUMEN}

Las redes universitarias corresponden al grado de evolución y de construcción académica de la institución universitaria de finales del siglo XX. En Colombia son de reciente creación con el objetivo de ofrecer en conjunto programas académicos de alto nivel como son los estudios de maestría y de doctorado. En este trabajo se realizará un balance histórico legal partiendo, de un primer período, del Decreto 080 de 1980 a la Constitución de 1991. El segundo período a partir de la Ley 30 de 1992 con el impacto de la misma en la construcción de Redes. Por su parte, los doctorados en educación, se crean con la peculiaridad organizativa de Redes académicas. En el caso de RUDECOLOMBIA, desde la construcción de la identidad de la Red, se proyecta con objetivos de desarrollo regional educativo y con proyectos de construcción de nación.

Palabras clave: Redes Universitarias, Universidad, Programas Académicos, Doctorados.

\footnotetext{
1 Doctora en Filosofía y Ciencias de la Educación, Profesora Titular de la Universidad Pedagógica y Tecnológica de Colombia. Organizó y fue la primera Directora del doctorado "Ciencias de la Educación" de RUDECOLOMBIA. Directora del Grupo de Investigación "Historia y Prospectiva de la Universidad Latinoamericana". HISULA.
} 


\title{
UNIVERSITY NETS IN COLOMBIA NEW HISTORICAL BACKGROUND FOR THE UNIVERSITY
}

\author{
Diana Elvira Soto Arango ${ }^{1}$ \\ Universidad Pedagógica y Tecnológica de Colombia - RUDECOLOMBIA \\ HISULA Research Group \\ dianaelvirasoto@gmail.com
}

\begin{abstract}
The university net belongs to the evolution and building academic in the University degree at the end of the twentieth century. In Colombia they are new; their aim is to offer a set of academic high level programs as Masters and Doctorates studies. In this work it will do a historical level balance starting in a first term, the decreto 080 of 1980 in the constitution. The second term, from the Law 30 of 1992 had the impact in the same Nets constructions. Thus, the education doctorates are created with the style of organization of academic nets. In this case RUDECOLOMBIA, since the first construction of the Net's is identity focus on objectives of regional education development ad with projects of nation construction.
\end{abstract}

Key words: University Nets, University, Academic Programs, Doctorates. 


\section{INTRODUCCIÓN}

La concepción de Red Universitaria está unida al desarrollo de la conceptualización de esta institución. En los inicios las "universitas" o "corporaciones" de estudiantes y profesores se caracterizaron por la internacionalidad de sus miembros y la identidad corporativa. Las universidades coloniales, del actual territorio colombiano, presentaron identidad corporativa que se trasladó a los núcleos de poder de la administración virreinal. La universidad en Colombia continúo con la identidad institucional, fundamentalmente en la universidad privada y en menor grado en la oficial. El gran salto con las redes universitarias se da al establecer una identidad de Red partiendo de la identidad institucional.

En el balance histórico legal se parte, de un primer período, cuando se establecen legalmente las redes universitarias en Colombia, dentro de la nueva concepción de universidad que se unía a la investigación, a partir del Decreto 080 de 1980 hasta la Constitución de 1991. El segundo período, a partir de la Ley 30 de 1992 con el impacto de la misma en la necesidad de presentar requisitos para la acreditación de programas de maestría y doctorado hecho que motivo la construcción de Redes académicas hasta el Decreto 1295 del 2010 que regula el Registro calificado y dentro de estos los programas en convenio.

En este trabajo conceptualizamos sobre la Red universitaria como una estructura organizativa que se auto regula y une voluntades académicas y financieras, bajo convenios, para lograr objetivos comunes principalmente de formación y de investigación bajo los valores de la solidaridad, respeto, tolerancia y corresponsabilidad sin depender de una institución en particular. ${ }^{2}$

Los principios fundantes de la Red los hemos tomado de RUDECOLOMBIA $^{3}$ y la dimensión de la Red está dada por el compromiso,

\footnotetext{
2 Las redes se caracterizan por la dimensión: espacial (definen espacios de acción), temporal (fijan tiempo de acción) y estructural (forma organizativa). ADLER LOMNITZ, Larissa. (2001): Redes sociales, cultura y poder. Ensayos de antropología latinoamericana, México: FLACSO.

3 SOTO ARANGO, Diana y LAGO CARAZO, Diana. (2009): "Fundamentos legales y epistémicos de RUDECOLOMBIA", Cartagena, 14 de febrero, p. 2.
} 
en la construcción de nación ${ }^{4}$ desde la región desde la perspectiva de trabajo en red en el contexto nacional e internacional. Nos limitaremos en el análisis de RUDECOLOMBIA sólo a la estructura de la Red de RUDECOLOMBIA por lo tanto no tomamos en el análisis el desempeño en el Doctorado en Ciencias de la Educación. En Colombia hemos localizado que las redes universitarias pueden categorizarse en: Académicas, de Investigación, Tecnológicas y de Servicios. Una Red puede ofrecer varias funciones de las señaladas.

Para entender las Redes universitarias en Colombia y la prospectiva de las mismas las debemos situar en el contexto socio-cultural de Colombia y Latinoamérica con problemas e ideales de transformación universitaria diferentes a otras regiones del mundo. Con esta línea de pensamiento surge la siguiente pregunta: ¿si las redes académicas universitarias han logrado una identidad de red que desde fuera impacten y transformen lo académico-investigativoadministrativo a las instituciones participantes y si están transformando la educación en los ámbitos regionales liderando políticas educativas en el contexto nacional?.

En definitiva, es preguntarnos desde las redes universitarias si éstas han logrado superar los imaginarios locales institucionales y si han logrado proyectarse con transformaciones en los contextos regionales, nacionales e internacionales.

El aporte de este trabajo es el de establecer un balance histórico-legal y de prospectiva, caracterizando las redes académicas y de investigación, con sus dificultades y aportes a un proceso de desarrollo del país, al tratar de construir nación desde los ámbitos regionales apoyándose en políticas educativas que exigen la organización de programas académicos como los doctorados que se presentan a la acreditación institucional de alta calidad académica apoyándose en las redes universitarias.

Los hechos históricos se enmarcan dentro del impacto externo e interno de

\footnotetext{
4 Tomamos en este trabajo la siguiente concepción de Nación "Es una comunidad política imaginada, construida, con forma histórica específica de organización social, y cohesión estatal como reflejo de la voluntad general", se sustenta de tres autores: Anderson, Eder y Francis. Para Anderson: "Nación es una comunidad política imaginada como inherentemente limitada y soberana." ANDERSON, Benedict. (2007): Comunidades imaginadas. Reflexiones sobre el origen y la difusión del nacionalismo. México, Fondo de Cultura económica, p. 93. Para Eder: es algo construido, simbólico". EDER, Klaus (2003): Identidades colectivas e mobilizacao de identidades, en Revista brasilera de Ciencias Sociales. Asociación Nacional de Pós-Graduacao e Pesquisa em Ciencias. Sao Paulo, p. 18. Para Francis “"Forma histórica específica de la organización social, que se da donde la mayoría de la población en un Estado moderno configura una unidad social, cuando dicha unidad se basa sobre todo en su cohesión estatal, y cuando el Estado es percibido como reflejo de la voluntad general". FRANCIS, Emerich. (1957): Wissenschaftliche Grundlagen soziologischen Denkens. Muchen,
} 
las redes que sustentan programas de doctorado. La metodología, con la cual se aborda este análisis, es el de la historia socio-política de la educación. Donde el hecho educativo de creación y evolución de las redes universitarias se estudia desde las relaciones político-académico-sociales. Las fuentes están dadas por la legislación colombiana; del SNIES, Gruplac, DocLac en COLCIENCIAS; "Informes del Viceministro de Educación, Documento del CNA," documentos de reuniones de Ministros de Educación en América Latina, y del archivo del Doctorado Ciencias de la Educación de RUDECOLOMBIA.

\section{Antecedentes históricos}

Las universidades, de occidente, se organizan dentro de una marcada territorialidad: Bolonia (corporación de estudiantes), ${ }^{6}$ París (corporación de maestros) ${ }^{7}$ en el siglo XII. Si bien existe la internacionalización de los estudiantes provenientes de diferentes lugares, se constituyeron por "naciones" según el lugar de origen, pero pronto la identidad ${ }^{8}$ hacia su corporación ${ }^{9}$ es el distintivo fundamental. Consideramos que desde esa identidad corporativa quizá como lo denomina Chiaramonte se establecieron y convivió la identidad de la "nación" interrelacionada con la identidad, hacia la corporación, en escala como las famosas "matrioshkales." 10 Estas corporaciones universitarias llegaron a

5 CNA. (2008): "Situación Actual de los Doctorados en Colombia: Análisis de Indicadores que tipifican características importantes”. Bogotá, abril del 2008, Documento de trabajo. Informe del Viceministro de Educación Superior, Sr. Gabriel Burgos Mantilla. Bogotá, abril.

6 Considera Le Goff que "en Bolonia se la primera originalidad: los profesores no forman parte de la universidad. La corporación universitaria sólo agrupa a estudiantes. Los profesores forman el Colegio de doctores". LE GOFF, Jacques. (1990): Los intelectuales de la edad media. Barcelona, GEDISA, p. 79.

7 Asociación de maestros e intelectuales que se reúnen con la intención de dedicarse a la enseñanza... Fueron los maestros los que establecieron las reglas y el canciller era uno de los maestros". CASTREJON DIEZ, Jaime. (1990): El concepto de universidad. México, Trillas, p. 107.

8 "Las identidades se construyen dentro, y no fuera, del discurso, tenemos que entender que están producidas a partir de estrategias enunciativas específicas, en ámbitos históricos institucionales específicos, en el seno de prácticas y formaciones discursivas específicas. Más aún, surgen dentro del juego de modalidades concretas de poder, de forma que son más el producto del señalamiento de la diferencia y la exclusión, que signos de una unidad idéntica naturalmente constituida". HALL, Stuart. (1996): "Introduction: Who Needs Identity?" en S. Hall \& P. Du Gay (eds.): Questions of Cultural Identity, Londres, SAGE, p. 4.

9 La Corporación "cuya finalidad es el monopolio local y que se beneficia ampliamente con los desarrollos nacionales o locales" en LE GOFF, Jacques. (1990): Op., cit, p. 77.

${ }^{10}$ Muñequitas rusas de albergar otra más pequeña en su interior y así sucesivamente. De este modo se constituyen series de identidades a diferente escala, que son no sólo compatibles sino que en ocasiones se refuerzan entre ŝ́". CAIRO CAROU, Heriberto. (2005): "Discursos geopolíticos y construcción de identidades supranacionales en América Latina", en RODRIGUES-MOURA Enrique, Von Wäldern, Städten und Grenzen: Narration und kulturelle Identitätsbildungsprozesse in: Lateinamerika, Anuario Jahrbuch des Österreichischen Lateinamerika-Instituts, número 8, Frankfurt am Main: Si utilizamos metafóricamente la capacidad de las conocidas Brandes \& Apsel / Südwind, pp.315-337. 
convertirse en el tercer poder político local a finales del siglo XII. ${ }^{11}$ Pero, reiteramos que lo relevante de estas primeras "corporaciones universitarias" es el carácter internacional de sus miembros que luego imprimían una identidad corporativa. Pero, esta internacionalización de sus miembros se perdió en París cuando el Rey, Luis XII en 1492, decidió convertir la universidad en una institución nacional. De esta manera, la "universitas" fue dejando el carácter internacional de sus miembros y dieron paso, a los estudiantes nacionales, con la identidad institucional que variaba según el amparo legal de la comunidad religiosa, Bula Papal, o secular por la Cédula Real.

Las instituciones que se fundaron con el título de universidad, en el territorio geográfico que hoy es Colombia, fueron Menores. ${ }^{12}$ Estas se resguardaron legalmente en una Bula Papal para otorgar los respectivos grados académicos a los juristas y sacerdotes. En la capital, Santa Fe, localizamos cuatro instituciones de este carácter con la categoría de Universidades Menores pero sólo dos otorgaban grados a laicos. Estas universidades pertenecían a comunidades religiosas donde no se establecía colaboración entre ellas, por el contrario, la rivalidad era relevante dado que los egresados formaban grupos de poder en la administración virreinal. ${ }^{13}$

La universidad Javeriana otorgó grados hasta 1767 y la de Santo Tomas hasta 1826. La única institución, en la Audiencia de Santafé, que otorgó grados y con administración del Cabildo Civil y que llevó Cédula Real fue el ColegioUniversidad de San Pedro Apóstol localizado en Mompox (1806). Por su parte el Colegio Mayor del Rosario, única institución con modelo salamantino y autonomía en el Claustro, no otorgó grados académicos. En definitiva, el legado de la universidad colonial fue el de identidad hacia la institución y/o comunidad religiosa según el caso. Sin embargo, hay que decir que llegamos a las universidades republicanas del siglo XIX y atravesamos casi todo el siglo XX sin advertir esa posibilidad que brinda la interrelación académica de las universidades y el trabajo en RED. Debemos indicar, que hacia los años setenta las reuniones de Ministros de Educación en Latinoamérica empezaron a plantear la necesidad de interrelacionar las universidades.

\footnotetext{
11 “El dominico Tomás de Irlanda escribe: "La ciudad de París es como Atenas, está dividida en tres partes: una es la de los mercaderes, de los artesanos y del pueblo que se llama la gran ciudad; otra es la de los nobles donde se encuentra la corte del rey y la iglesia catedral y que se llama la Cité; la tercera la de los estudiantes y de los colegios que se llama universidad" (siglo XIII). LE GOFF, Jacques. (1990): óp., cit, p. 77.

12 SOTO ARANGO, Diana. (2005): "Aproximación histórica a la Universidad Colombiana”, en Revista Historia de la Educación Latinoamericana, Tunja, $N^{\circ} 7$, Tunja, Universidad Pedagógica y Tecnológica de Colombia, RUDECOLOMBIA, p.104.

${ }^{13}$ A finales del siglo XVIII con el grupo de criollos ilustrados que se vinculó a la Expedición Botánica se dio un paso de integración intelectual.
} 
Nosotros consideramos que las redes académicas y de investigación deben estar sustentadas en una nueva concepción de universidad. ${ }^{14}$ Los principios fundantes de la Red los hemos tomado de RUDECOLOMBIA ${ }^{15}$ que se respaldan en la CORRESPONSABILIDAD ${ }^{16}$ enmarcada en la autonomía universitaria responsable; la integración desde la complementariedad y la sinergia; transparencia y confiabilidad; la alteralidad en el respeto, comprensión y ética en la relación de cooperación. La dimensión de la Red universitaria está dada por el compromiso, en la construcción de nación ${ }^{17}$ desde la región, formando y consolidando comunidades académicas regionales que propongan desarrollos y alternativas de programas académicos de alto nivel, que impacten la calidad de vida de la sociedad colombiana, a partir de una convivencia de equidad y justicia social desde la perspectiva de trabajo en red en el contexto nacional e internacional. $^{18}$

Las Redes universitarias las hemos clasificado según la función que realizan. La primera hace referencia a la Red Académica, se presenta en una estructura organizativa que en convenio reúne varias instituciones para ofrecer uno o varios programas académicos, no dependen de ninguna institución, por ejemplo, RUDECOLOMBIA.

La segunda, la Red de Investigación se caracteriza por la estructura organizativa que, se autorregula, reúne varios grupos de investigación bajo un

\footnotetext{
${ }^{14}$ La universidad la entendemos como "la comunidad académica de estudiantes y profesores reunidos en una institución para establecer un diálogo, interno y externo, crítico teórico-práctico en un ambiente de libertad que promueve la esencia de su ser: la investigación científica interdisciplinar que genera "bienes públicos" que modifican los parámetros de la realidad establecida en un contexto cultural, socio-político y económico específico con visión internacional", SOTO ARANGO, Diana. (2009): "Los doctorados en Colombia", en Revista Historia de la Educación Latinoamericana, $N^{\circ} 13$, Tunja, Universidad Pedagógica y Tecnológica de Colombia, RUDECOLOMBIA, p. 166.

15 RUDECOLOMBIA. (2009): "Manifiesto de rectores de RUDECOLOMBIA sobre Redes universitarias". Cartagena, 14 de febrero del 2009," en Revista Historia de la Educación Latinoamericana, Nùmero 12, Tunja, Universidad Pedagógica y Tecnológica de Colombia, RUDECOLOMBIA, pp.286-287. SOTO ARANGO, Diana y LAGO CARAZO, Diana. (2009): "Fundamentos legales y epistémicos de RUDECOLOMBIA", óp., cit.

${ }^{16}$ Corresponsabilidad: "Compromiso de cada universidad en particular desde su autonomía y del colectivo en RED. Es la responsabilidad compartida frente a unos intereses comunes sustentados en la confianza y en la igualdad de derechos y deberes. SOTO ARANGO, Diana y LAGO CARAZO, Diana. (2009): "Fundamentos legales y epistémicos de RUDECOLOMBIA", Doc. Cit., p.1.

${ }^{17}$ Se localiza el Centro de investigación "VENDIMIA" que tiene como objetivo la investigación sobre "la construcción de nación en Latinoamérica y el Caribe". Vendimia, la lidero en su organización el Grupo de investigación: "Historia y prospectiva de la Universidad Latinoamericana". HISULA. en el año 2006. Cada año se reúne y edita la publicación "Cuadernos de Vendimia".

18 SOTO ARANGO, Diana y LAGO CARAZO, Diana. (2009): Doc., cit., p. 1.
} 
programa común de búsqueda que se desarrolla de manera interdisciplinaria y transdiciplinaria. No dependen de ninguna institución y tienen como valor central la solidaridad, el respeto y la tolerancia, por ejemplo VENDIMIA. El máximo desarrollo de una Red de investigación pertenece a los Centros de Excelencia. ${ }^{19}$

La tercera, corresponde a las Redes tecnológicas ${ }^{20}$ que presentan estructuras organizativas, bajo convenio, tienen como objetivo central desarrollar asistencias tecnológicas y de medios virtuales. Generalmente, tienen apoyos gubernamentales. En Colombia, la mayoría de las instituciones pertenecen a RENATA. La cuarta corresponde a la Red de servicios, con estructura organizativa propia, sustentada en convenios, que tiene como finalidad prestar servicios de diferente índole a las instituciones. Una Red puede ofrecer más de una función de las señaladas.

\section{La legislación sobre Redes Universitarias en Colombia}

La posibilidad legal de establecer la interrelación académica en las universidades colombianas se da sólo a partir del Decreto 080 de 1980. Este Decreto establece los requisitos que debe tener un establecimiento de educación superior $^{21}$ y define estrategias para una nueva concepción sobre la universidad.

Es relevante conocer que la Educación Superior antes olvidada, en las prioridades educativas de las políticas gubernamentales, se va trasladando a un primer plano para obtener un mayor control sobre la misma. Desde luego era necesario "acomodar" la universidad a los avances tecnológicos". En definitiva, debía ingresar a la globalización del conocimiento. En tal sentido, el presidente Turbay Ayala se trazó la tarea de reformar la universidad y como hemos indicado expidió el Decreto 80 de 1980 con las facultades extraordinarias que le concedía

\footnotetext{
${ }^{19}$ COLCIENCIAS lo define como: "Los Centros de Investigación de Excelencia son, de acuerdo con el Sistema Nacional de Ciencia y Tecnología, una red nacional de grupos de investigación articulada alrededor de un programa común de trabajo en un área científica y tecnológica considerada como estratégica para el país. Como criterios básicos para la creación de estos centros se tiene en cuenta el impacto socioeconómico nacional, el dinamismo de las actividades seleccionadas y las externalidades asociadas, la capacidad de científica, la capacidad para captar recursos de cooperación internacional, la capacidad de formar doctores y facilitar la formación posdoctoral además de consolidar alianzas estratégicas con pares internacionales de investigación”.

${ }^{20}$ GALINDO, Jesús. (2001): "Redes y comunidades virtuales. La construcción social en el mundo, el ciberespacio y el hipermundo", tomado desde internet (online) el 13/ 02/2010 en: www.geocities.com/arewara/arewara.htm

${ }^{21}$ DECRETO 080 de 1980, En el art. 1: define los principios y fija las normas que regulan la Educación Post-secundaria o Superior.
} 
el "Estado de Sitio". Este Decreto establece que la universidad es un "servicio público bajo el control del Estado" (Art. 2). De esta manera, se introduce la función de la investigación en las instituciones universitarias y establece la relación entre la investigación y la docencia (Art. 4). Como es de suponer se conceptualiza la universidad con una nueva mirada: la investigación. ${ }^{22}$ Precisamente, es este concepto de "investigación" el que va a permitir dentro de la "sociedad del conocimiento" que esta actividad no se puede realizar en solitario por una persona o institución.

Asimismo, unido a este concepto de universidad investigativa, se instaura los estudios de postgrado ${ }^{23}$ que se les designa de "formación avanzada" y clasifica en: académica o de especialización. ${ }^{24}$ Es importante indicar que se determina que los estudios de formación académica "son aquellos en los que prevalece la investigación y prepara a la persona para la actividad científica. Ellos deben cumplirse con criterio interdisciplinario" (Art. 35). Debemos indicar que estos estudios de formación avanzada son los que van a originar las primeras redes universitarias académicas.

Pero, es precisamente dentro de la nueva concepción de universidad, que el citado Decreto 080, introduce las Redes académicas al indicar que "Las instituciones de Educación Superior podrán celebrar convenios interinstitucionales con el fin de lograr una óptima utilización de sus recursos. Con el mismo fin, el Gobierno Nacional podrá autorizar a las instituciones oficiales la celebración de contratos y convenios con otras instituciones educativas y con las no educativas" (Art. 177).

Sin embargo, como hemos indicado, la realidad universitaria de 400 años de identidad corporativa, especialmente en aquellas de mayor tradición como Javeriana, Santo Tomás, Rosario y últimamente Andes, ${ }^{25}$ entre otras, que han

\footnotetext{
${ }^{22}$ DECRETO 80 de 1980, la Universidad se define como: "la entidad que tenga aprobados al menos tres programas de formación Universitaria en diferentes áreas del conocimiento y acredite una significativa actividad de investigación y suficientes y adecuados recursos humanos y físicos. Está reservado a estas instituciones el empleo de la denominación de Universidad".

23 "Estos se habían iniciado en la Universidad Nacional en el año 1949 donde se otorgaba el título de especialista. La expansión de estos estudios se da a partir de los años sesenta en las universidades públicas y privadas". Véase en SOTO ARANGO, Diana. (1990): "La reforma universitaria de los postgrados en Colombia". En 14 Congreso de la CESE. Reformas e innovaciones educativas en el umbral del siglo XXI: Una perspectiva comparada. Madrid, España, 2 - 7 de julio, 1990. p. 1.

${ }^{24}$ DECRETO 080 del 22 de enero de 1980. Art. 34: "El máximo nivel de la educación superior lo constituye la modalidad de formación avanzada y tiene por objeto la preparación para la investigación y para la actividad científica o para la especialización. La investigación constituye el ámbito necesario de esta modalidad"

${ }^{25}$ Se crea en 1949.
} 
establecido núcleos de poder, desde la colonia, a través de la ubicación de sus egresados, en cargos que van desde la Presidencia de la República, Ministerios y empresas que llevan con presunción el pertenecer a su institución universitaria. Estas barreras difíciles de romper se encontraron con la circunstancia que si no se unían con otras instituciones no podían desarrollar la investigación función que ya asumía la universidad en el contexto internacional y en el país de forma obligatoria para los programas de doctorado.

Hay que decir, que Colombia sufre un cambio sustancial en las instituciones a partir de la Constitución de 1991 y dentro de estas la universidad. Por este motivo, se expide la Ley 30 de 28 de diciembre de $1992 .{ }^{26}$ Esta Ley, amplía el concepto de universidad dentro de los parámetros de la globalización del conocimiento y la investigación científica. Es relevante que aparecen las propuestas de los organismos supranacionales, nacionales, instituciones universitarias y asociaciones para hacerle frente a los cambios de la "sociedad de la información" que ha transformado los estudios superiores. En tal sentido es importante resaltar que la UNESCO adoptó el término y concepto de "sociedades del saber" que permiten la dimensión de la transformación cultural, socio-política-económica e institucional.

Sin duda alguna, América Latina se vio abocada a dar un cambio en los estudios universitarios y especialmente en los de postgrado. La teoría de los "recursos humanos" 27 reforzó el papel asignado a la universidad al considerar la educación como un factor de desarrollo. Es así, que la universidad forma y en concreto en el doctorado a las personas para desempeñarse en actividades especializadas dentro de las "exigencias de la modernización." 28 Aún hoy, este criterio prevalece debido a la competencia laboral; a la dificultad de estudiar fuera del país; y al estar fuera del mercado de los estudios científicos capaces de generar nuevas tecnologías e innovaciones en las diferentes áreas de la ciencia. Y en efecto, los estudios doctorales, se orientaron primordialmente a dar movilidad social o profesional.

Pero, reiteramos que es precisamente, a partir de la Ley 30 de $1992,{ }^{29}$ que se inicia el proceso de ubicar la universidad dentro del contexto de la

\footnotetext{
${ }^{26}$ La LEY 30 del 28 de diciembre de 1992, por el cual se organiza el servicio público de la educación superior. Publicado por PACHECO, Iván. (2001): Nuevo Compendio de Normas sobre la Educación Superior. Bogotá, ICFES, Art. 19, p. 19

${ }^{27}$ OROZCO, Luis Enrique. (1987): "La autonomía interna como fundamento de la relación con el estado: el caso colombiano"; en Universidad y política en América Latina, México, Universidad Nacional Autónoma de México, p.64.

${ }^{28}$ Ibídem., p. 64

${ }^{29}$ Se establece la Reforma Educativa.
} 
internacionalización del conocimiento y de la ciencia. ${ }^{30}$ La preocupación central se centró en impulsar una universidad vinculada a la investigación, el hacerla más concordante con los avances de la ciencia pero especialmente, el ubicar la institución en la nueva era predominante de las reformas universitarias en América Latina. ${ }^{31}$ Pero es necesario valorar en su justa medida que es bien diferente la Ley a la aplicación de la misma donde se encuentra con una realidad de crisis económica especialmente en las universidades públicas.

Pero, ciertamente, es importante un elemento que va hacer pensar a las universidades en la necesidad de establecer interrelación a través de redes universitarias y es el hecho de la acreditación institucional y de programas. Evidentemente, la citada Ley 30 crea el Consejo Nacional de Educación Superior -CESU- que tiene como tarea fundamental, proponer políticas y planes que sean conducentes para el desarrollo de la Educación Superior, reglamentaciones, procedimientos y mecanismos para la evaluación de la calidad de la educación que impartan las instituciones de Educación Superior. ${ }^{32}$ Por otro lado se establece en el artículo 81 que se crea el sistema Universidades del Estado, integrado por las universidades oficiales y cuyos objetivos se orientan: a)."Racionalizar y optimizar los recursos humanos, físicos, técnicos y financieros; b).Implementar la transferencia de estudiantes, el intercambio de docentes, la creación o fusión de programas académicos y de investigación, la creación de programas académicos conjuntos".

A tenor de este artículo 81 se deduce que la política nacional se encaminaba al apoyo de las redes universitarias como medio para poder acceder a las acreditaciones nacionales e internacionales que empezaban hacer carrera en el ámbito internacional. Lo esencial es que se estaba legislando en el año 1992 para 30 universidades oficiales y 43 privadas. ${ }^{33}$

Por otra parte, la "Misión de sabios: al filo de la oportunidad", presenta su informe el 24 de julio de 1994, y en las recomendaciones indican que: "Las universidades que adelanten programas de doctorado se articularán en una Red de programas de investigación de excelencia, de tal manera que cada

\footnotetext{
${ }^{30}$ SOTO ARANGO, Diana. (2005): “Aproximación histórica a la Universidad Colombiana", Op., cit, pp. 99 - 136.

${ }^{31}$ Véase un amplio estudio sobre las reformas universitarias del Siglo XX en América Latina en: SOTO ARANGO, Diana, JARDILINO, José Rubens. (2006): Políticas universitarias en América Latina. Sao Paulo. Brasil, Tomo III Colección Historia y prospectiva de la universidad latinoamericana. UNINOVE, Universidad Pedagógica y Tecnológica de Colombia, RUDECOLOMBIA, p. 242.

${ }^{32}$ LEY 30 de 1992, Art. 34, 35 y 36. Se crea el Consejo Nacional de Educación Superior - CESU.

${ }^{33}$ Fuente: Estadísticas del ICFES, 2002.
} 
programa doctoral de la red tenga una rigurosa acreditación nacional, cuente con estudiantes becados de tiempo completo para la totalidad del ciclo y se realice en cooperación con otras instituciones nacionales y con una universidad extranjera de reconocido prestigio." 34

Hay que decir, que en los inicios de los años noventa se inician las reuniones para crear las redes académicas entre universidades oficiales del Estado Colombiano y en este lapso de tiempo se crean, entre otras, las dos Redes de los doctorados en Educación en los años $1993^{35}$ y $1996 .{ }^{36}$

La legislación colombiana nuevamente regula las redes académicas dentro del Decreto 916 de 22 mayo del 2001 que unificaba los requisitos y procedimientos para los programas de Maestría y Doctorado. Se indica que "En la autorización de programas ofrecidos en Convenio con instituciones extranjeras, la Comisión Nacional de Maestrías y Doctorados evaluará la contribución efectiva que dicho convenio implique para el programa en términos de calidad"37

Precisamente, al plantear los términos de calidad, en el año 2003 el Decreto 2566 establece que estos programas de convenios deben obtener el Registro Calificado. Se indica que para la "Apertura de programas en CONVENIO: cuando dos o más instituciones de educación superior decidan ofrecer un programa académico en convenio, este deberá surtir el trámite señalado en este decreto para la obtención del Registro calificado"38 y "La solicitud del Registro calificado para ofrecer y desarrollar un programa académico en convenio entre instituciones de educación superior deberá realizarse conjuntamente por los rectores o representantes legales de las instituciones de educación superior que lo suscriban." ${ }^{39}$

\footnotetext{
34 "Misión de los sabios. Colombia: al filo de la oportunidad", Bogotá, 21 de julio de 1994. TERCER MUNDO EDITORES. 1996, "Colección documentos de la Misión". Tomo 1. Informe conjunto de Eduardo Aldana Valdés, Luis Fernando Chaparro Osorio, Gabriel García Márquez, Rodrigo, Eduardo Posada Flórez, Ángela Restrepo Moreno, Carlos Eduardo Vasco Uribe, Carlos Eduardo Vasco - Comisionado coordinador.

${ }^{35}$ Convenio firmado Número 139 de 1993, mediante el cual las universidades Pedagógica Nacional, Valle, Antioquia, Industrial de Santander y Nacional, se comprometieron a organizar el doctorado en Educación, que fue aprobado por el Ministerio de Educación Nacional de Colombia mediante la Resolución 1991 de mayo 16 de 1996

${ }^{36}$ Convenio Interinstitucional del 4 de junio de 1996, aprobó el Doctorado en Ciencias de la Educación el Ministerio de Educación Nacional de Colombia por Resolución 2333 del 3 de julio del 1998 .

${ }^{37}$ DECRETO 916 de 22 mayo del 2001, Art. 17. Parágrafo.

${ }^{38}$ DECRETO 2566 de 9 octubre de 2003, Art. 27.

${ }^{39}$ DECRETO 2566 de 9 octubre de 2003, Art. 28.
} 
Tres años después, mediante el Decreto 1001 del 3 de abril del 2006, se organiza la oferta de los programas de posgrado y para los programas en Convenio se agrega que cuando se realiza con instituciones internacionales el título lo otorga la Universidad colombiana. ${ }^{40}$ No deja de ser significativo que en la Ley 1188 del 2008 no se nombren las redes académicas ni los convenios. Hecho similar se encuentra en el Documento reglamentario. Hay que decir, que se presenta una tendencia en algunos sectores académicos por demeritar la actividad de las Redes académicas. Sin embargo, están cambiando estos procesos y mentalidades y el Ministerio de Educación Nacional de Colombia, en el año 2010 reglamenta en el Decreto1295 ${ }^{41}$, a través de los artículos 26 a 29 los Programas en Convenio para obtener el Registro Calificado.

\section{Caracterización de las redes actuales}

Las redes universitarias en América Latina tienen mayor sentido por las dificultades económicas y la falta de recursos humanos en las universidades para adelantar programas de alta calidad académica. La IX Cumbre Iberoamericana de Jefes de Estado y de Gobierno" que reunió a 21 países Iberoamericanos en la ciudad de la Habana, Cuba, el 16 de noviembre de 1999 estableció apoyar 15 programas y proyectos. Dentro de los cuales se encuentra el "programa de cooperación en el desarrollo de programas de doctorado y en la dirección de Tesis doctorales." 42

Así mismo, en la reunión del CRES, que se realizó en Cartagena del 4 al 7 de junio del 2008, estableció en las conclusiones finales la relevancia y el apoyo que se le debería prestar a las Redes académicas. En este punto fue significativa la intervención del Dr. Luis Enrique Arango, presidente de RUDECOLOMBIA.

Reiteramos que las Redes de universidades, para ofrecer programas de doctorado con calidad académica, han sido la única alternativa posible para las universidades del Estado ubicadas en las diferentes regiones del país con desarrollos educativos desiguales. Como hemos indicado las Redes de los programas de doctorado en educación fueron pioneros en esta modalidad organizativa. En el primer caso, la Red de las denominadas "cinco grandes universidades" o iglesias mayores como las denomina el Dr. Guillermo Hoyos:

\footnotetext{
40 DECRETO 1001 del 3 de abril del 2006Capítulo V. De los convenios entre instituciones de educación superior. Art.9. facultad para ofrecer posgrados en convenio con instituciones nacionales y extranjeras. El titulo lo dará la institución colombiana".

${ }^{41}$ DECRETO 1295 del 20 de abril del 2010, en el capítulo VIII referente a "Convenios para el desarrollo de programas académicos", y la RESOLUCIÓN 5443 del 30 de junio del 2010, este último para programas de educación.

42 Publicado en: Revista Iberoamericana de Educación, Madrid, OEI, p.117.
} 
Pedagógica Nacional, Valle, Antioquia, Industrial de Santander y Nacional firmaron el Convenio del año 1993 y les aprobaron el programa en Educación en el año 1996. Estas universidades abrieron el programa académico en tres de las cinco instituciones. En el año 2000 se presentaron a la Acreditación previa, parece ser, que al considerarse cada una con gran fortaleza los criterios de RED decayeron y no superaron la acreditación de calidad con excepción de la Universidad de Antioquia.

La segunda Red denominada RUDECOLOMBIA, ${ }^{43}$ y como las identifica el Dr. Hoyos "iglesias menores" corresponden a las universidades de: Atlántico, ${ }^{44}$ Cauca, Caldas, Cauca, Cartagena, Nariño, Quindío, ${ }^{45}$ Pedagógica y Tecnológica de Colombia, Tecnológica de Pereira, Tolima. Lo anterior, identifica el carácter interinstitucional de las diez universidades citadas que están ubicadas en la región norte, centro y sur del país. Es importante conocer que tres de las universidades mencionadas se originaron a comienzos de la República, ${ }^{46}$ una en los albores del siglo $\mathrm{XX}^{47}$ y el resto pertenecen a la época de mediados del siglo $\mathrm{XX}{ }^{48}$ Esta Red firmó el primer convenio el 4 de junio de 1996, el programa del Doctorado en Ciencias de la Educación se aprobó en el año 1998 y obtuvo la acreditación previa en el año 2000. En el 2005 obtuvo el Registro calificado y en el 2008 el premio a la calidad de la Asociación Iberoamericana de Postgrados. AUIP. En el 2010 se presento a la renovación del Registro calificado y en prospectiva para el 2011 aspira a presentarse al CNA para la acreditación de alta calidad.

Consideramos, que esta red ha demostrado que cuando existen voluntades políticas y académicas y se unen debilidades y fortalezas se pueden lograr programas de alta calidad académica. En noviembre del 2010, el Doctorado Ciencias de la Educación de RUDECOLOMBIA presentaba 214 estudiantes, 52 graduados. Los estudiantes son atendidos por 114 profesores de planta con 79 grupos clasificados en COLCIENCIAS. ${ }^{49}$ Las universidades de la RED

\footnotetext{
${ }^{43}$ La condición de Red se ha legitimado mediante convenios celebrados entre las Universidades de RUDECOLOMBIA así: 4 de Junio de 1996, 17 de diciembre de 1996, 4 de julio de 1997, 21 de septiembre de 1998, 28 de octubre de 1998; 3 de marzo del 2000, 16 de julio y 18 de diciembre del 2005, 3 de junio del 2008, 17 de noviembre del 2009, a partir de los cuales se ha definido el marco general para el funcionamiento del Doctorado en Ciencias de la Educación, producto de la experiencia adquirida en la cooperación y en las sucesivas reformas educativas de carácter nacional.

${ }^{44}$ Esta universidad ingreso en el año 1999

45 Esta universidad ingreso en el año 2008

${ }^{46}$ En 1827 las universidades de: Cauca, Cartagena, y Pedagógica y Tecnológica de Colombia.

${ }^{47}$ La universidad de Nariño en 1904.

48 Caldas (1943), Tolima (1954) y Tecnológica de Pereira (1958).

${ }^{49}$ Circular 42 del año 2008 de la Directora Académica, Los datos corresponden al Comité Curricular celebrado el 11 de agosto del 2008.
} 
tienen una cobertura de casi 165.000 mil estudiantes que son asistidos por 6.500 profesores de planta y un número mayor de docentes por contrato. Todas tienen un mínimo de tres facultades. Las ciudades donde están localizadas las universidades están entre los 200.000 a 1.500 .000 mil habitantes. ${ }^{50}$

\section{Balance de una Red Académica Universitaria}

Este balance se presenta desde dos miradas diferentes. El primero, se relaciona con los aportes que confiere la RED a cada Universidad y el segundo, con los aportes que otorga la Universidad a la RED.

En cuanto a los aportes de la RED a cada institución podemos destacar los siguientes tomados de la experiencia de RUDECOLOMBIA ${ }^{51}$ :

\section{Procesos académicos}

En primera instancia destacamos la participación de los profesores en una comunidad académica de doctores desempeñándose como investigadores, profesores de curso, Tutores, Directores de Tesis y Jurados evaluadores. ${ }^{52}$

Segundo, participación en grupos de investigación y proyectos conjuntos con la respectiva socialización de los resultados en la comunidad académica nacional en Simposios, reuniones, congresos

Tercero, en la participación de un proyecto editorial y producción científica de impacto nacional e internacional.

Cuarto, la construcción de un modelo asociativo académico.

Quinto, se constituyeron nuevos ambientes académicos favorables para la creación de nuevos programas de doctorado.

Sexto, el programa de Doctorado en Ciencias de la Educación se ha

\footnotetext{
${ }^{50}$ Popayán con 258.653; Cartagena con 899.200; Manizales con 388.525; Pasto con 416.842; Ibagué con 511.600; Pereira con 428.397, Tunja con 351.082 habitantes, Barranquilla con 1.182.493.

${ }^{51}$ Síntesis desarrollada en conjunto con la Dra. Lago y Dr. Arango en la reunión del día jueves 12 de noviembre del 2009 en Villa de Leyva durante la celebración del III Seminario Internacional de VENDIMIA.

${ }^{52}$ En el caso de RUDECOLOMBIA para todos los profesores fue su primera experiencia de docentes en cursos de doctorado con la excepción de Diana Soto Arango, quien había dictado cursos de doctorado en la UNED de España.
}

Rev. hist. edu. latinoam. Vol. 15. Año 2010, pp. 241 - 270 
convertido en cada institución en el referente y sustento en las acreditaciones de las instituciones de la Red.

\section{Internacionalización}

En este sentido priorizamos los 62 convenios firmados, de ellos 43 vigentes, que han permitido la movilidad y las becas de los estudiantes y profesores, investigaciones y publicaciones conjuntas.

En efecto la movilidad se ha concretado en relación con los de profesores en las pasantías académicas, investigaciones conjuntas y participación en eventos. Igualmente, la movilidad de los estudiantes se ha evidenciado en las pasantías académicas, en un $90 \%$ con becas que otorga la universidad extranjera. La movilidad de los directivos ha facilitado escenarios para la firma de los convenios. Hemos podido comprobar que la forma mas eficiente de una Misión académica es cuando se vinculan los tres estamentos de la RED.

De forma paralela, en este contexto se destaca el trabajo de los grupos de investigación con otros grupos de diferentes países que posibilita desarrollo de investigaciones, publicaciones y eventos en conjunto.

\section{Visibilidad de las instituciones a través de la RED}

Adquiere importancia el hecho de la visibilidad en el contexto nacional e internacional de la imagen corporativa e institucional de la RED a través de: eventos, publicaciones, Web (en el caso de RUDECOLOMBIA desde el $2007)^{53}$, otras Web. ${ }^{54}$

\section{Formación de profesores doctores para cada institución}

Efectivamente, se creó la necesidad formar una comunidad crítica y se puso en evidencia el requerimiento de tener doctores en cada institución. Por otro lado, se ha dado reconocimiento a la condición inherente al desempeño de un doctor

\footnotetext{
53 Visitas Totales Web de RUDECOLOMBIA: de Septiembre 2007 a Noviembre de 2009: 77.218 Visitas. 70,29\% de Nuevas Visitas. 29.71\% de Usuarios Comunes

${ }^{54}$ Actualmente, se mide la visibilidad de las universidades iberoamericanas a través de las Web. Véase en www.webometrics.inf TOP 6000 Universities, TOP Latín América dentro de 17.000 universidades la Universidad de Sao Paulo ocupa el primer lugar en Latinoamericana y el 34 en el nivel mundial seguida de la UNAM para Latinoamérica y el 44 en el contexto internacional. De las universidades Colombianas la Universidad de los Andes ocupa el 20 lugar en Latinoamérica y el 459 en el contexto mundial seguida de la Universidad Nacional de Colombia en expuesto 26 en Latinoamérica y el 541 en el contexto mundial.
} 
dentro del proceso académico-investigativo, al dársele espacios académicos en la institución y en la Red para su desarrollo como investigador autónomo.

\section{Construcción de un proyecto nacional}

Hemos pretendido rescatar en este apartado la integración de los rectores de RUDECOLOMBIA en una solidaridad política-administrativa que se evidencia en los diferentes contextos de las reuniones de la educación superior nacional. Encontramos también que la relación de las universidades de las diferentes regiones del país (norte, centro y sur) permite a los rectores, docentes, estudiantes y egresados estar permanentemente en contacto con la realidad regional de Colombia.

En tal sentido, situaríamos el impacto regional, del modelo asociativo, tomado de RUDECOLOMBIA y que se ha aplicado en la organización de otras Redes universitarias académicas como la del SUE-Caribe. ALMAMATER. Por ultimo, destacaríamos la identidad hacia la RED, en un proceso de construcción y que fue valorada positivamente por los pares internacionales de la Asociación Universitaria Iberoamericana de Postgrados (AUIP) en el 2007.

\section{Impacto administrativo-académico en la normatividad institucional}

Por lo que hemos podido analizar en cada una de las universidades de RUDECOLOMBIA se ha visto que el programa de Doctorado Ciencias de la Educación influenció directamente los cambios en los Reglamentos académicos y de postgrado de cada institución, debido a que anteriormente no se incluía el nivel de doctorado en los mismos.

No es un hecho aparte que se crearon en conjunto los nuevos programas de Maestría en Educación desde el Comité Curricular y de auto-evaluación de RUDECOLOMBIA. Este modelo curricular y Plan de estudios se llevo a cada una de las universidades donde no existía este programa académico, promoviendo la articulación de la Maestría con el doctorado en Ciencias de la Educación.

\section{Una estructura académico-administrativa-financiera}

En el caso de RUDECOLOMBIA ha logrado en estos trece años organizar una estructura sólida de funcionamiento académico a través de un Consejo de Rectores, el Comité Nacional de Currículo y de auto evaluación ${ }^{55}$ y los Comités

\footnotetext{
${ }^{55}$ Denominación que se le otorgó a este Comité desde febrero del 2005 por medio de oficio del Presidente de RUDECOLOMBIA.
} 
académicos de doctorado en cada universidad. El modelo de organización administrativa ha logrado dinamizar la Red con el elemento prioritario de agilidad e información permanente a través de Circulares que se realizan desde la Presidencia y Dirección Académica Nacional. En este contexto se debe inscribir, el aspecto financiero, dentro un proceso de aprendizaje que se logro solucionar en el 2005 a través de Alma Mater.

En definitiva, consideramos que la Red es la estructura académicoadministrativo que permite llevar los programas de doctorados y de maestría de cada institución a la excelencia académica.

Por otra parte, el Impacto de cada Universidad en la Red se puede establecer a través de:

La confianza que depositan los rectores al dar viabilidad al proyecto, constituyendo el primer convenio interinstitucional. En el caso de RUDECOLOMBIA, el liderazgo ejercido por el rector de la Universidad Pedagógica y Tecnológica de Colombia, Dr. Carlos Sandoval. Destacamos, la generosidad de los Presidentes de RUDECOLOMBIA, en especial al Dr. Luis Enrique Arango, bajo la presidencia de él se iniciaron los programas de doble titulación.

Por otra parte, el compromiso y confianza en la sostenibilidad de los principios asociativos que ha viabilizado la Red como soporte del Doctorado Ciencias de la Educación. Asimismo, el aporte presupuestal de cada universidad al programa evidente en: dedicación de profesores doctores; infraestructura física y dotación de los CADES; la cuota anual de cada universidad que permite el desarrollo de las actividades administrativas de la RED.

En lugar destacado, situaremos el compromiso y liderazgo de los docentes doctores como esencia de los programas doctorales. A partir de ellos, se inicia el trabajo académico-investigativo colectivo de la Red.

Asimismo, la logística institucional que permite el desarrollo de reuniones y eventos en las diferentes universidades. Hay que decir, que nos encontramos en proceso de aprendizaje institucional para que los Convenios Internacionales que gestione cada universidad con los contactos académicos se amplían a la Red. Igualmente, el lograr que la Red se haga visible al interior de las universidades, en la región y en el extranjero, desde una política institucional con proyección social. 


\section{Caracterización de RUDECOLOMBIA. Modelo de integración universitaria para América Latina}

Sin lugar a dudas, la experiencia de RUDECOLOMBIA se pone en la palestra con un resultado exitoso de cooperación inter-universitaria. La Red ha logrado superar los escenarios de los cambios administrativos y las complejas demandas de los entornos político-regionales que afectan a cada una de las instituciones y los obstáculos que interfieren en el ejercicio pleno de la autonomía universitaria frente a los sectores políticos regionales. El gran reto que teníamos de organizar, un programa académico, en cada una de las universidades fundadoras de la Red ya se cumplió. En tal sentido, las nuevas universidades que ingresaron en el 2006 y 2007 han culminado su proceso de organización académica y esperamos el respectivo registro para iniciar labores en el año 2011.

Pero si hubiera que subrayar lo más significativo de la Red de RUDECOLOMBIA es su identidad, con la misma, construida a través de estos catorce años que expresa la voluntad de un grupo comprometido con unos ideales que desarrollan sus propias dinámicas incidiendo en su entorno institucional, local y regional. Consideramos que desde esa identidad local quizá como lo denomina Chiaramonte se ha establecido una serie de identidades a diferente escala como las famosas "matrioshkales" 56 . Es allí desde esas identidades de RED, universidad, región, que se desarrolla el proyecto del Centro de Investigación de VENDIMIA ${ }^{57}$ de la construcción de nación ${ }^{58}$ desde la región colombiana. En tal sentido, lo relevante es permitir desarrollar esos valores de identidad y que no seamos unos "pachuchos" como les pasa a los mexicanos que viven en Norteamérica que bien analiza Octavio Paz en su libro "El laberinto de la soledad" 59 . Reiteramos, los egresados ${ }^{60}$ de RUDECOLOMBIA dan cuenta del trabajo de la identidad con la Red y el programa académico.

\footnotetext{
${ }^{56}$ Indicado al comienzo de este trabajo en la cita 10.

${ }^{57}$ Este proyecto se lidera desde VENDIMIA con el proyecto marco "Educación, cultura política e interculturalidad en las regiones y en los proyectos de nación en Colombia. Siglos XVIII a XXI". Vendimia es la unión de grupos de investigación que, en el año 2006, realizaron una unión temporal para participar en la convocatoria de COLCIENCIAS. Esta no la obtuvieron pero los grupos continuaron con el proyecto y a la fecha han realizado cuatro seminarios internacionales y un libro producto de los dos primeros eventos académicos.

${ }^{58}$ No vamos a centrarnos en la gran discusión teórica que sobre el concepto de nación se ha planteados desde el siglo XIX. Nosotros retomando a varios teóricos consideramos que es"la autoconstrucción de identidades" partiendo del reconocimiento de una historia conjunta como lo analizamos en la cita 4 de este trabajo.

59 PAZ, Octavio. (1991): El Laberinto de la Soledad. México, Fondo de Cultura Económica.

${ }^{60}$ En la VII Reunión de los egresados de RUDECOLOMBIA, se constituyeron en Asociación, en la reunión celebrada en Villa de Leyva, el día 12 de noviembre del 2009.
} 
Por su puesto construir identidades no es tarea fácil en las redes académicas cuando se mueven intereses institucionales y regionales. Esta es una labor que se establece con la generosidad "de ayudar al más débil"; de valorar y dar sentido a la colaboración académica del "otro". Contra las Redes el gran enemigo son los imaginarios de las instituciones que consideran que "uno es más que dos" y que la individualidad es más relevante en los procesos académicos. En tal sentido, refuerza este imaginario la política de financiación de COLCIENCIAS a los programas de doctorado y la falta de una reglamentación al Registro de Redes académicas por parte del Ministerio de Educación Nacional.

Como hemos indicado este doctorado de RUDECOLOMBIA se desarrolla en Ciencias de la Educación. Se inició con las áreas de formación en: Historia de la Educación Latinoamericana. Posteriormente, se organizaron las de Pedagogía Currículo y Didáctica, y Pensamiento Pedagógico y Comunicación. Actualmente, cuenta con seis líneas de formación ${ }^{61}$ que se trabajan desde los grupos de investigación. De todas maneras se debe tener presente que este doctorado surgió de una Red de Historiadores de la Educación Latinoamericana que en 1992 realizaron su primer Congreso en Santafé de Bogotá. Una de las conclusiones, de este evento, fue el de organizar un doctorado en esta área del conocimiento. Y, en este caso se demuestra la influencia internacional que el programa ha tenido desde los inicios al contar con el apoyo de la Sociedad de Historia de la Educación Latinoamericana. SHELA

RUDECOLOMBIA, ${ }^{62}$ en su calidad de Red universitaria académicoinvestigativa se caracteriza por:

${ }^{61}$ Acta del Comité Curricular y de Auto-evaluación de octubre 17 a19 de 2008, reunión celebrada en la Universidad del Cauca.

\begin{tabular}{|l|l|l|}
\hline \multicolumn{1}{|c|}{$\begin{array}{c}\text { AREAS DE } \\
\text { CONOCIMIENTO }\end{array}$} & \multicolumn{1}{|c|}{$\begin{array}{c}\text { LÍNEAS DE } \\
\text { FORMACIÓN }\end{array}$} & $\begin{array}{l}\text { UNIVERSIDADES DE LA RED } \\
\text { ADSCRITAS }\end{array}$ \\
\hline Historia de la educación & $\begin{array}{l}\text { Historia social y cultural. } \\
\text { Educación superior y Universidad }\end{array}$ & $\begin{array}{l}\text { Cartagena, Tolima, UPTC, } \\
\text { Nariño, UTP }\end{array}$ \\
\hline $\begin{array}{l}\text { Pedagogía Currículo y } \\
\text { Didáctica }\end{array}$ & Didáctica de las Ciencias \\
$\begin{array}{l}\text { Historia de la educación y } \\
\text { Pedagogía Currículo y } \\
\text { Didáctica }\end{array}$ & $\begin{array}{l}\text { Formación y desarrollo de } \\
\text { educadores }\end{array}$ & $\begin{array}{l}\text { Cartagena, Caldas, UPTC y } \\
\text { atlántico, Quindío }\end{array}$ \\
\hline Comunicación & $\begin{array}{l}\text { Comunicación, lenguaje, educación, } \\
\text { cultura }\end{array}$ & $\begin{array}{l}\text { Cauca, UTP, UPTC, Quindío, } \\
\text { Nariño }\end{array}$ \\
\hline Pedagogía Social & Pedagogía- educación social & $\begin{array}{l}\text { Tolima, UTP, Nariño, Magdalena, } \\
\text { Atlántico, Cartagena, UPTC }\end{array}$ \\
\hline $\begin{array}{l}\text { Pedagogía Currículo y } \\
\text { Didáctica }\end{array}$ & $\begin{array}{l}\text { Mediaciones tecnológicas y } \\
\text { ambientes virtuales }\end{array}$ & $\begin{array}{l}\text { Caldas, UPTC, UTP, Atlántico, } \\
\text { Quindío }\end{array}$ \\
\hline
\end{tabular}

${ }^{62}$ RUDECOLOMBIA representa la unión de voluntades académicas y administrativas de diez universidades regionales de Estado colombiano para lograr el desarrollo del Doctorado en Ciencias de la Educación. 
1. El carácter interinstitucional de las diez instituciones citadas, ubicadas en la región norte, centro y sur del país. La estrategia metodológica de la organización interna de RUDECOLOMBIA se sustenta en una estructura legal de convenios con Reglamento general y específicos y una estructura académica-administrativa-financiera interinstitucional. La dinámica de la Red se relaciona directamente con el trabajo coordinado y conjunto de la Red en unidad de voluntades que se establece a través de:

a.Reglamentos internos dirigidos al funcionamiento de la Red y del programa que se tiene en conjunto.

b.Dirección general colegiada en el Consejo Rectores, máximo organismo, integrado por los rectores de la RED bajo la dirección de un Presidente; El Comité Curricular integrado por un representante académico de cada universidad y un director académico nacional. Estos consejos se caracterizan por la participación, la coordinación, la cooperación y la concertación

c.Coordinaciones académicas múltiples. Cada universidad tiene un organismo académico con un modelo autonómico que planifica pero coordinadamente con el Comité Curricular maximizando los recursos financieros y humanos.

2.La administración financiera la realiza otra Red que se denomina ALMA MATER ${ }^{63}$ La Red se auto-financia con cuotas de cada universidad. Cada CADE tiene autonomía financiera con el ingreso del dinero de las matrículas.

3. La Internacionalización. La Red se apoya a través de convenios que han permitido desarrollar el intercambio de sus miembros y vincularlos a Redes Internacionales; participar en Congresos Internacionales, coordinados por RUDECOLOMBIA o invitados especiales en Coordinaciones de Simposios; las posibilidades de publicar en revistas Indexadas y en colecciones acreditadas internacionalmente; la posibilidad de asistir a cursos impartidos entre profesores nacionales e internacionales; la presencia de jurados internacionales en la sustentación de tesis doctorales. El programa participa y lidera Redes Internacionales. Destacamos por su entidad las "Misiones académicas". La Red desde los inicios ha utilizado como mecanismo, de internacionalización del programa, el sistema de la "Misiones académicas al exterior". Las "Misiones académicas al exterior" se han entendido como la actividad académica que

\footnotetext{
${ }^{63}$ Esta Red se creó por las universidades del "eje cafetero" en Colombia. En el año 2000.
} 
realizan en conjunto: profesores, estudiantes y directivos de RUDECOLOMBIA en varias instituciones de un país o países en el exterior. En definitiva, el programa de doctorado que se dirige sustenta su calidad académica en las posibilidades internacionales que le brinda la RED.

4.Auto-evaluación con auto-regulación. Estas apuntan directamente a las estrategias y políticas de la Red. La mirada permanente de la historia y la autoevaluación de la Red ha permitido aprovechar los éxitos y revisar las dificultades conceptuales, teóricas, logísticas y organizativas. La auto-regulación nos ha permitido, a su vez, aclarar y profundizar sobre aspectos relevantes de la organización administrativa y el desarrollo académico del programa que dirige la Red. Se establece prospectiva desde una visión concertada en la misión que permite tener la capacidad para entender y formular con anticipación las tendencias educativas que se avecinan.

5.Flexibilidad de la Red. Las Instituciones que componen la Red tienen un funcionamiento sustentado en la autonomía universitaria. Estas instituciones son reguladas por Leyes y Decretos Nacionales y Acuerdos y resoluciones de la Institución. Por lo tanto, la RED respeta esta dinámica interna de la universidad y sólo unificar en el funcionamiento académico los elementos propios que le da la Ley y Decretos Nacionales.

6.Los productos de la Red de RUDECOLOMBIA se concentran actualmente en la formación doctoral en un programa piloto en Ciencias de la Educación. Este programa se caracteriza por:

a) Flexibilidad e interdisciplinariedad curricular. Los estudiantes pueden recibir los cursos en cualquiera de las sedes del doctorado y/o universidades extranjeras con las que se tenga convenio. Desarrollan pasantías nacionales. El Plan de estudios actual, del Doctorado en Ciencias de la Educación, se presenta como la continuidad de la maestría y se sustenta en tres componentes de 80 créditos discriminados así: a). Núcleos Básicos del Saber Pedagógico (15\%); b). Fundamental Disciplinar (20\%); d). Producción y desarrollo de investigación. Tutorías, Grupos de Investigación, Seminario de Tesis (65\%). Actualmente, presenta seis líneas de formación doctoral.

b) Modelo educativo centrado en el estudiante. Quizá el modelo de la Tutoría sea novedoso en la interrelación que se da entre tutor, CADE y el Codirector de Tesis Internacional. A partir, del examen de suficiencia el estudiante es asesorado por el Director de Tesis Nacional (que puede continuar el mismo Tutor si es especialista en el tema), el Codirector de Tesis Internacional interviene 
en la planeación de la pasantía internacional del estudiante. Hasta el momento han salido en pasantía con beca 75 estudiantes. Un aspecto a resaltar es la identidad del estudiante con la Red.

c).Investigativo e interdisciplinario sustentado en 79 grupos de investigación con clasificados en COLCIENCIAS. Estos grupos tienen una dinámica propia en eventos y publicaciones que fortalecen la actividad académica del doctorado con un modelo interdisciplinario que se aplica a la investigación y a la estructura curricular. Ha generado nuevas competencias en la formación investigativa y desarrollado nuevas propuestas como el Centro de Investigación «VENDIMIA» y el Postdoctorado. Las Publicaciones son producto de los proyectos que desarrollan los grupos de investigación.

d) Auto-evaluación permanente Interna y externa. Por este motivo el programa se ha llevado a cuatro evaluaciones desde su corta creación hace doce años. Esta función la ejerce el Comité Curricular donde se detecta los problemas y se le establece de inmediato la solución pertinente llevándola a reglamentaciones que se dan a conocer por medio de las Circulares de la Dirección Académica. Hemos indicado que hemos traspasado 4 evaluaciones. Que el Doctorado en Ciencias de la Educación ha establecido su modelo de evaluación en 13 factores que fueron ponderados en marzo del 2007. En el año 2008 obtuvo el Premio de la calidad que otorga la Asociación Iberoamericana de Postgrados. AUIP.

e) Integración de los egresados en los procesos de: auto-evaluación, académicos, investigativos y en los eventos. Presencia de los 52 egresados que están liderando investigaciones nacionales e internacionales. Igualmente, están dirigiendo programas académicos de doctorado, son asesores del Ministerio de Educación Nacional y pioneros en nuevas líneas de investigación en el país. Se ha desarrollado estrategias para vincularlos a las actividades de investigación, académicas y de extensión del doctorado. Se destaca la participación de los mismos en las publicaciones y Misiones académicas de RUDECOLOMBIA.

f). Impacto regional y nacional, a través del establecimiento de una Red de doctores; Desarrollo académico institucional; Desarrollo regional educativo a través del Doctorado en Ciencias de la Educación. Esta política se ha cristalizado en el desarrollo de investigaciones y asesorías a instituciones educativas, entre otras, las Escuelas Normales. Se hace necesario que señalemos, especialmente, la relevancia que tiene un doctorado en una universidad en la región en que este ubicada. Por todos es conocido que una 
universidad regional se convierte en el núcleo cultural más importante de la provincia y de la ciudad.

7.La visibilidad de la Red se da a través de sus productos: Programas académicos, publicaciones y eventos. Los medios que se están utilizando son: la web de RUDECOLOMBIA, prensa y TV local y regional. Los espacios de comunicación nacionales y latinoamericanos están en construcción.

8. Impacto de la Red se manifiesta a través de los cambios de mentalidad institucional; y el desarrollo institucional y regional educativo.

9.Impacto de cada Universidad en la Red. primera instancia destacamos la confianza que depositaron los rectores fundadores en la viabilidad del proyecto, constituyendo el primer convenio, como alianza interinstitucional. En el caso de RUDECOLOMBIA, el liderazgo ejercido por el rector de la Universidad Pedagógica y Tecnológica de Colombia, Dr. Carlos Sandoval. El compromiso y confianza en la sostenibilidad de los principios asociativos que ha viabilizado la Red como soporte del Doctorado Ciencias de la Educación. Asimismo, el aporte presupuestal de cada universidad al programa evidente en: dedicación de profesores doctores; infraestructura física y dotación de los CADES; la cuota anual de cada universidad que permite el desarrollo de las actividades administrativas de la RED.

En lugar destacado, situaremos el compromiso y liderazgo de los docentes doctores como esencia de los programas doctorales. A partir de ellos, se inicia el trabajo académico-investigativo colectivo de la Red.

Por otra parte, la logística institucional que permite el desarrollo de reuniones y eventos en las diferentes universidades. Hay que decir, que nos encontramos en proceso de aprendizaje institucional para que los Convenios Internacionales que realice cada universidad y contactos académicos que se amplían a la Red. Igualmente, el lograr que la Red se haga visible al interior de las universidades, en la región y en el extranjero, desde una política institucional.

10. La Prospectiva de RUDECOLOMBIA. Consideramos que RUDECOLOMBIA dentro de la dinámica del funcionamiento de la RED, replantea la transformación universitaria desde nuevas concepciones del trabajo académico que está unido a la "sociedad del conocimiento". En el siglo XXI no se puede concebir un trabajo académico en solitario, por lo menos para las universidades de menor desarrollo investigativo como son las latinoamericanas. 
RUDECOLOMBIA, desde la Visión del Doctorado en Ciencias de la Educación "En el próximo quinquenio el Doctorado en Ciencias de la Educación tendrá reconocimiento nacional e internacional por su excelente nivel de desarrollo investigativo y de publicaciones indexadas, por ser interlocutor válido en las políticas públicas de educación, por la consolidación de la comunidad académica e investigativa y por su compromiso con la formación de educadores en el contexto del proyecto de construcción de nación". Este programa fortalecerá el Centro de investigación VENDIMIA como uno de los medios de cualificación de los grupos de investigación. De esta manera cumpliría los objetivos de una Red de investigación.

Una meta para el próximo año es la de la acreditación de alta calidad en Colombia, hecho que ya logró en el contexto Iberoamericano. El fortalecimiento se debe dar a través de la internacionalización en los convenios transversales que permitan el desarrollo de sus docentes a través de los proyectos de investigación con Pares Internacionales. La organización de Centros de Excelencia con el trabajo unificado de los grupos, ejemplo el Centro de investigación VENDIMIA. Las publicaciones deben ser la prioridad dada la característica académica de la Red ubicando el sello editorial de la misma. Las publicaciones deben estar marcadas por la calidad editorial y académica garantizada por los evaluadores nacionales e internacionales.

Finalmente, consideramos que está RED de universidades deberá dar el siguiente paso para establecer otros programas en otras áreas del conocimiento

que retomen la experiencia de este primer programa académico del Doctorado Ciencias de la Educación.

\section{CONCLUSIÓN}

Las redes universitarias corresponden al grado de evolución y de construcción académica de la institución universitaria de finales del siglo XX. En Colombia son de reciente creación con el objetivo de ofrecer en conjunto programas académicos de alto nivel como son los estudios de maestría y de doctorado. La legislación colombiana permitió la conformación de las Redes universitarias a partir del Decreto 080 de 1980, con una nueva concepción de universidad para optimizar recursos y ofrecer programas académicos de alta calidad. Sin embargo la última Ley de educación, 1188 del 2008, no las nombra ni reglamenta respecto a los programas que coordinan y a su modus operandi en: títulos y Registros.

Sin lugar a dudas, la experiencia de RUDECOLOMBIA se pone en la 
palestra con un resultado exitoso de cooperación universitaria en la categoría de Red académica. La Red ha logrado superar los escenarios de los cambios administrativos y las complejas demandas de los entornos político-regionales que afectan a cada una de las instituciones y la débil autonomía universitaria frente a los sectores políticos regionales.

Pero si hubiera que subrayar lo más significativo de la Red de RUDECOLOMBIA, en su calidad de sustento administrativo para el programa doctoral en Ciencias de la Educación, habría que referirse a la experiencia adquirida a través de estos trece años que expresa la voluntad de un grupo comprometido con unos ideales que desarrollan sus propias dinámicas incidiendo en su entorno institucional, local y regional. Con los grupos de investigación se ha presentado un impacto nacional e internacional. Pero hay otra distinción que es la mirada permanente de la historia de la red y la auto-evaluación que ha permitido beneficiar los éxitos y revisar las dificultades conceptuales, teóricas, logísticas y organizativas. La auto-regulación nos ha concedido, a su vez, aclarar y profundizar sobre aspectos relevantes de desarrollo académico

Si bien, la realidad no se desconoce. Colombia es un país en guerra donde la inversión en educación superior es un problema secundario. Desde esta perspectiva puede parecer que una Red, universitaria académica, con el objetivo de formar doctores en educación no tenga relevancia cuando las políticas gubernamentales se inclinan hacia el apoyo de lo tecnológico. No desconocemos las demandas actuales de la denominada "triada ciencia -tecnología-empresa" que incide en la financiación de los programas de investigación. Pero la pregunta que surge es si ¿Colombia, en los actuales momentos, tiene los medios y el dinero para suministrar ciencia y tecnología? Si ¿los doctorados tienen que orientarse en esta vía? O por el contrario, damos prioridad a tratar de resolver el problema central de la falta de valores en la educación que transformen las estructuras mentales de la población colombiana.

En cualquier caso, el reto para RUDECOLOMBIA está planteado: realizar transformaciones en la universidad y a su entorno apoyado en la Red. Desde allí se trazara la política de transformación para que la universidad colombiana no sea simplemente como la definía Unamuno "una oficina del Estado, con su correspondiente expediente didáctico" ${ }^{64}$ sino por el contrario se presente como una institución donde "la comunidad académica de estudiantes y profesores desarrollen un diálogo, interno y externo, crítico, teórico-práctico en un ambiente

\footnotetext{
${ }^{64}$ UNAMUNO, Miguel de. (1889):"De la enseñanza superior en España", en: Revista
} Nueva, Madrid, p. 19. 
de libertad que promueve la esencia de su ser: la investigación científica interdisciplinar que genera "bienes públicos" que modifican los parámetros de la realidad establecida en un contexto cultural, socio-político y económico específico con visión internacional”.

En definitiva, RUDECOLOMBIA organizada como estrategia de apoyo académico de universidades oficiales regionales para desarrollar las políticas gubernamentales de "acreditación institucional" ha marcado derroteros de organización administrativa-académica. Consideramos que se ha construido una cultura de identidad de Red donde la cooperación académica liderada por los rectores, docentes y estudiantes de RUDECOLOMBIA presentan un modelo de diálogo y respeto a la diversidad cultural de cada institución; las relaciones financieras sólidas; el modelo curricular y la creación de una comunidad académica que se ha fortalecido con las relaciones Internacionales. Finalmente, consideramos que esta "memoria" del desarrollo de RUDECOLOMBIA nos permite desde la investigación histórica analizar "la razón de su identidad y el valor de futuro de la memoria institucional" 65 que nos lleva a una nueva concepción de universidad.

\title{
FUENTES
}

\author{
ARCHIVOS \\ ARCHIVOGENERALDELANACIÓNDECOLOMBIA(AGN) \\ ARCHIVORUDECOLOMBIA. Sede Universidad Pedagógica y Tecnológica de Colombia. UPTC \\ ARCHIVORUDECOLOMBIA. Sede Central en Bogotá. \\ ARCHIVOPERSONALDIANASOTOARANGO. FondoEducación. RUDECOLOMBIA
}

\section{LEGISLACIÓN}

Decreto 80 del 22 de enero 1980. Art. 134 y 177

Decreto 916 de 22 mayo del 2001. Art. 17

Decreto 2566 de 9 octubre de 2003. Art. 27 y 28

Decreto 1001 del 3 de abril del 2006. Cap. V Art. 9

DECRETO 1295 del 20 de abril del 2010

RESOLUCIÓN 5443 del 30 de junio del 2010

Convenios de RUDECOLOMBIA

INSTITUTO COLOMBIANO FOMENTO DE LA EDUCACIÓN SUPERIOR. ICFES,

Estadísticas de la Educación Superior. Bogotá. Sub-dirección de Planeación del ICFES.

Ley 30 de 1992.

\footnotetext{
65 ESCOLANO BENITO, Agustín. (1998): "sobre el oficio del maestro y los programas de formación. Nuevos enfoques genealógicos", en La Universidad del Siglo XX. España e Iberoamérica. Murcia, Universidad de Murcia, p. 680.
} 


\begin{tabular}{|c|c|}
\hline Año & Instituciones, Leyes Decreto \\
\hline \multirow[t]{3}{*}{1980} & Período: 1980 a 1991 \\
\hline & $\begin{array}{l}\text { Decreto } 80 \text { del } 22 \text { de enero } 1980 \text {. Este decreto establece los requisitos que debe tener } \\
\text { un establecimiento de educación superior. La universidad se concibe dentro de } \\
\text { parámetros de investigación. Art. } 4 \text {. Investigación y docencia; Art. 31. "titulo en la } \\
\text { respectiva disciplina" (se elimina los títulos de doctor a las profesiones). Artículo } 47 . \\
\text { Sólo puede obtener su reconocimiento institucional como universidad la entidad que } \\
\text { tenga aprobados al menos tres programas de Formación Universitaria en diferentes } \\
\text { áreas del conocimiento y acredite una significativa actividad de investigación y suficientes } \\
\text { y adecuados recursos humanos y físicos. Está reservado a estas instituciones el empleo } \\
\text { de la denominación de Universidad. } \\
\text { REDES: Artículo 134. El Gobierno Nacional podrá autorizar a las instituciones } \\
\text { oficiales de Educación Superior para participar en la constitución de empresas } \\
\text { industriales y comerciales del Estado y sociedades de economía mixta, para un mejor } \\
\text { uso de sus recursos. Artículo 177. Las instituciones de Educación Superior podrán } \\
\text { celebrar convenios interinstitucionales con el fin de lograr una óptima utilización de } \\
\text { sus recursos. Con el mismo fin, el Gobierno Nacional podrá autorizar a las instituciones } \\
\text { oficiales la celebración de contratos y convenios con otras instituciones educativas y } \\
\text { con las no educativas. }\end{array}$ \\
\hline & \\
\hline 1992 & $\begin{array}{l}28 \text { de diciembre Ley } 30 \text {. Se organiza el servicio público de la Educación Superior.".Art. } \\
19 \text {."Son universidades las reconocidas actualmente como tales y las instituciones } \\
\text { que acrediten su desempeño como criterio de universalidad en las siguientes } \\
\text { actividades: La investigación científica y tecnológica, la formación académica en } \\
\text { profesiones o disciplinas y la producción desarrollo y transmisión del conocimiento y } \\
\text { de la cultura universal y nacional. Estas instituciones están igualmente facultadas } \\
\text { para adelantar programas de formación en ocupaciones, profesiones o disciplinas, } \\
\text { programas de especialización, maestrías, doctorados y postdoctorados, de conformidad } \\
\text { a la presente Ley" (Art. 19) }{ }^{66} \text {. } \\
\text { REDES: Art. 81.Se crea el sistema Universidades del Estado, integrado por las } \\
\text { universidades oficiales objetivos: a)."Racionalizar y optimizar los recursos humanos, } \\
\text { físicos, técnicos y financieros; b).Implementar la transferencia de estudiantes, el } \\
\text { intercambio de docentes, la creación o fusión de programas académicos y de } \\
\text { investigación, la creación de programas académicos conjuntos. }\end{array}$ \\
\hline 2001 & $\begin{array}{l}\text { Decreto } 916 \text { de } 22 \text { mayo del } 2001 \text {.Se unifican requisitos y procedimientos para los } \\
\text { programas de Maestría y Doctorado. (A este lo deroga el Decreto } 1001 \text { del } 3 \text { de abril } \\
\text { del } 2006 \text {. Se organiza la oferta de los programas de postrado) } \\
\text { REDES. CONVENIOS INTERNACIONALES Art. 17. Parágrafo "En la autorización } \\
\text { de programas ofrecidos en Convenio con instituciones extranjeras, la Comisión Nacional } \\
\text { de Maestrías y Doctorados evaluará la contribución efectiva que dicho convenio } \\
\text { implique para el programa en términos de calidad". }\end{array}$ \\
\hline
\end{tabular}

${ }^{66}$ La ley 30 del 28 de diciembre de 1992, por el cual se organiza el servicio público de la educación superior. Publicado por PACHECO, Iván (2001): Nuevo Compendio de Normas sobre la Educación Superior. Bogotá, ICFES, Art. 19, p. 19. 


\begin{tabular}{|c|l|}
\hline Año & \multicolumn{1}{|c|}{ Instituciones, Leyes Decreto } \\
\hline 2003 & $\begin{array}{l}\text { DECRETO 2566 de 9 octubre de 2003.Establece las condiciones mínima de calidad y } \\
\text { demás requisitos para el ofrecimiento y desarrollo de programas académicos de educación } \\
\text { superior y se dictan otras disposiciones. } \\
\text { REDES: Art. 27. "Apertura de programas en CONVENIO: cuando dos o más } \\
\text { instituciones de educación superior decidan ofrecer un programa académico en convenio, } \\
\text { este deberá surtir el trámite señalado en este decreto para la obtención del Registro } \\
\text { calificado" } \\
\text { Art. 28: De los convenios para ofrecer y desarrollar programas } \\
\text { Parágrafo 2: "La solicitud del Registro calificado para ofrecer y desarrollar un programa } \\
\text { académico en convenio entre instituciones de educación superior deberá realizarse } \\
\text { conjuntamente por los rectores o representantes legales de las instituciones de educación } \\
\text { superior que lo suscriban". }\end{array}$ \\
\hline 2006 & $\begin{array}{l}\text { Decreto 1001 del3 de abrildel 2006... Se organiza la oferta de los programas de postgrado. } \\
\text { REDES. Capítulo V. De los convenios entre instituciones de educación superior. Art.9. } \\
\text { facultad para ofrecer postgrados en convenio con instituciones nacionales y extranjeras. } \\
\text { El titulo lo dará la institución colombiana. Art. 10. Registro calificado la evaluación de } \\
\text { manera integral. Art. Condiciones mínimas: }\end{array}$ \\
\hline 2008 & $\begin{array}{l}\text { Ley 1188 del 25 de abril del 2008. Por el cual se regula el registro calificado de los } \\
\text { programas de educación superior. Art. 2: Condiciones de calidad para el Registro. Art. 5. } \\
\text { Introduce los ciclos propedéuticos. Deroga el Art. 1 del Decreto 2566 del 2003. }\end{array}$ \\
\hline 2009 & $\begin{array}{l}\text { En el concepto de universidad se continua con lo establecido en la Ley 30 de 1992, art. 19. } \\
\text {-El concepto de doctorado, Decreto 1001 del 3 de abril del 2006. Art. 7 (no menciona el } \\
\text { postdoctorado que se había señalado Decreto 916 de 22 mayo del 2001.) } \\
\text { Registro Calificado: Ley 1188 del 25 de abril del 2008. Por el cual se regula el registro } \\
\text { calificado de los programas de educación superior. }\end{array}$ \\
$\begin{array}{l}\text { Decreto 1295 del 20 de abril del 2010. Art. 26 a 29. Registro para Programas en } \\
\text { Convenio. Art. 26. Conceptualización sobre los doctorados. }\end{array}$ \\
\hline
\end{tabular}

\section{REFERENCIAS}

ADLER LOMNITZ, Larissa. (2001): Redes sociales, cultura y poder. Ensayos de antropología latinoamericana, México: FLACSO.

CASTREJON DIEZ, Jaime. (1990): El concepto de universidad. México, Trillas.

CAIRO CAROU, Heriberto. (2005): "Discursos geopolíticos y construcción de identidades supranacionales en América Latina”, en RODRIGUES-MOURA Enrique Von Wäldern, Städten und Grenzen: Narration und kulturelle Identitätsbildungsprozesse in Lateinamerika, (Anuario Jahrbuch des Österreichischen Lateinamerika-Instituts, número 8), Frankfurt am Main: Si utilizamos metafóricamente la capacidad de las conocidas Brandes \& Apsel / Südwind.

CNA. (2008): "Situación Actual de los Doctorados en Colombia: Análisis de Indicadores que tipifican características importantes". Bogotá, abril del 2008, Documento de trabajo. Informe del Viceministro de Educación Superior, Sr. Gabriel Burgos Mantilla. Bogotá, abril.

DABAS, Elina. (1993): Red de redes. Las prácticas de intervención en redes sociales, Buenos Aires, Paidós.

DABAS, Elina y NAJMANOVICH, Denise. (1995): Redes. El lenguaje de los vínculos. Hacia la reconstrucción y el fortalecimiento de la sociedad civil, Buenos Aires, Paidós. 
ESCOLANO BENITO, Agustín. (1998): "sobre el oficio del maestro y los programas de formación. Nuevos enfoques genealógicos”, en La Universidad del Siglo XX. España e Iberoamérica. Murcia, Universidad de Murcia.

GALINDO, Jesús (2001) "Redes y comunidades virtuales. La construcción social en el mundo, el ciberespacio y el hipermundo". Artículo en línea, disponible en http://www.geocities.com/arewara/ arewara.htm

HALL, Stuart. (1996): “Introduction: Who Needs Identity?” en S. Hall \& P. Du Gay eds.): Questions of Cultural Identity, Londres, SAGE.

LE GOFF, Jacques. (1990). Los intelectuales de la edad media. Barcelona, GEDISA.

OROZCO, Luis Enrique. (1987): "La autonomía interna como fundamento de la relación con el estado: el caso colombiano"; en Universidad y política en América Latina, México, Universidad Nacional Autónoma de México.

PACHECO, Iván. (2001): Nuevo Compendio de Normas sobre la Educación Superior. Bogotá, ICFES.

SOTO ARANGO, Diana y LAGO CARAZO, Diana. (2009): "Fundamentos legales y epistémicos de RUDECOLOMBIA", Cartagena, 14 de febrero.

SOTO ARANGO, Diana. (1990): "La reforma universitaria de los postgrados en Colombia". En 14 Congreso de la CESE. Reformas e innovaciones educativas en el umbral del siglo XXI: Una perspectiva comparada. Madrid, España, 2 - 7 de julio.

SOTO ARANGO, Diana. (2005): “Aproximación histórica a la Universidad Colombiana”, en Revista Historia de la Educación Latinoamericana, Tunja, $N^{\circ} 7$, Tunja, Universidad Pedagógica y Tecnológica de Colombia, RUDECOLOMBIA.

SOTO ARANGO, Diana, JARDILINO, José Rubens. (2006): Políticas universitarias en América Latina. Sao Paulo. Brasil, Tomo III Colección Historia y prospectiva de la universidad latinoamericana. UNINOVE, Universidad Pedagógica y Tecnológica de Colombia, RUDECOLOMBIA.

SOTO ARANGO, Diana. (2009): "Los Doctorados en Colombia. Un Camino hacia la Transformación Universitaria" en Revista Historia de la Educación Latinoamericana No 12, Tunja, Universidad Pedagógica y Tecnológica de Colombia, RUDECOLOMBIA.

DE UNAMUNO, Miguel. (1889):'De la enseñanza superior en España”, en Revista Nueva, Madrid.

VASCO, Carlos Eduardo y otros. (1996): "Misión de los sabios. Colombia: al filo de la oportunidad", Bogotá, 21 de julio de 1994. Tercer Mundo Editores.

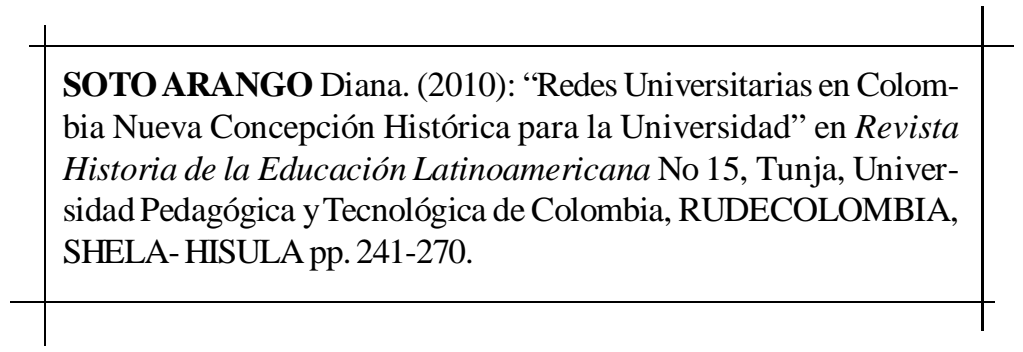

Rev. hist. edu. latinoam. Vol. 15. Año 2010, pp. 241 - 270 